Document downloaded from:

http://hdl.handle.net/10251/84387

This paper must be cited as:

Torregrosa, AJ.; Broatch Jacobi, JA. (2016). Compact High-Pressure Intake Silencer with Multilayer Porous Material. SAE International Journal of Passenger Cars. Mechanical Systems. 9(3):1-8. doi:10.4271/2016-01-1819.

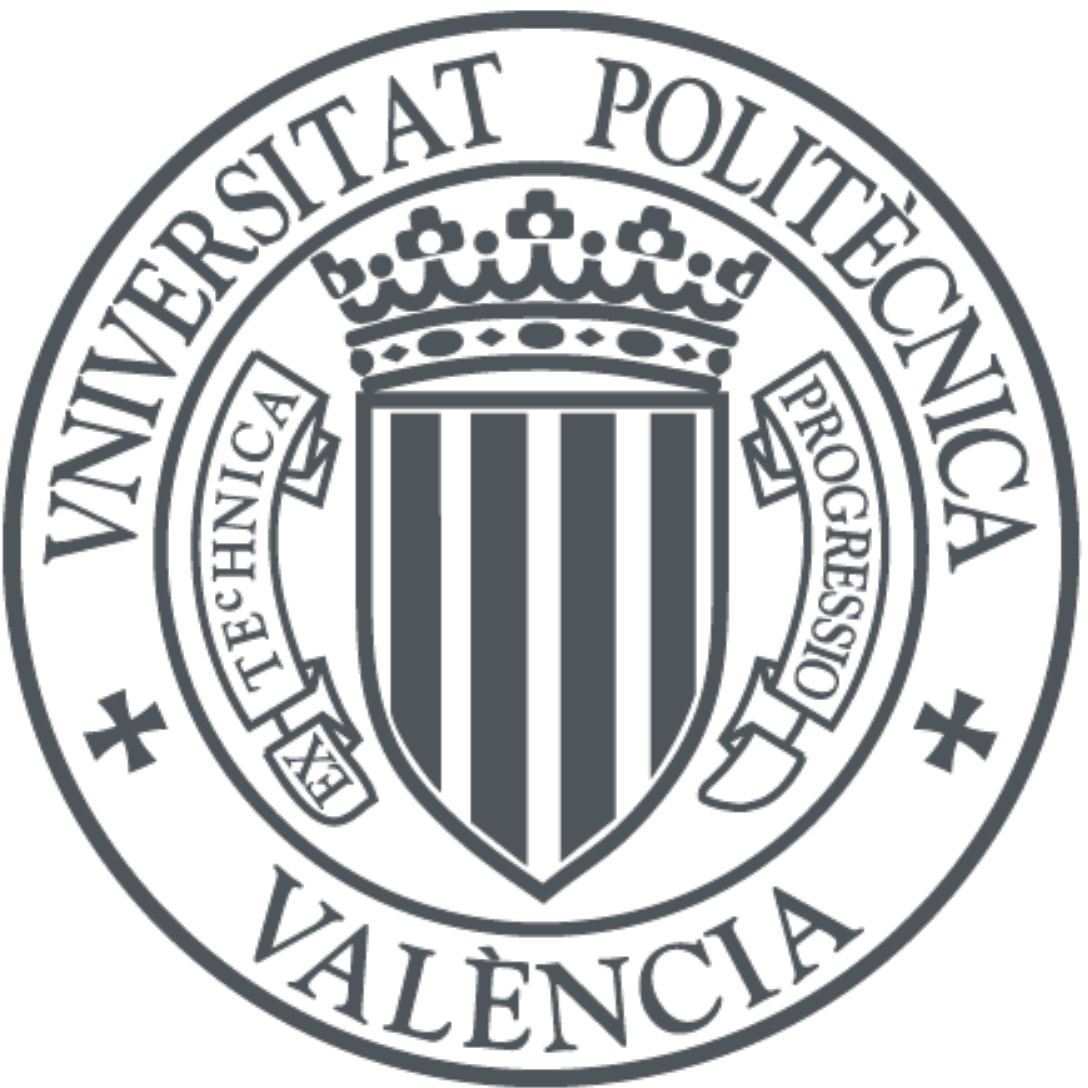

The final publication is available at

http://dx.doi.org/10.4271/2016-01-1819

Copyright

Additional Information 


\title{
Compact High-Pressure Intake Silencer with Multilayer Porous Material
}

\author{
A. J. Torregrosa, A. Broatch \\ CMT - Motores Térmicos. Universitat Politècnica de València \\ V. Raimbault, J. Migaud \\ Mann Hummel France
}

\begin{abstract}
Intake noise has become one the main concerns in the design of highly-supercharged downsized engines, which are expected to play a significant role in the upcoming years. Apart from the low frequencies associated with engine breathing, in these engines other frequency bands are also relevant which are related to the turbocharger operation, and which may radiate from the highpressure side from the compressor outlet to the charge air cooler. Medium frequencies may be controlled with the use of different typologies of resonators, but these are not so effective for relatively high frequencies. In this paper, the potential of the use of multi-layer porous materials to control those high frequencies is explored. The material sheets are located in the side chamber of an otherwise conventional resonator, thus providing a compact, lightweight and convenient arrangement. Several configurations have been tested in an impulse rig, without and with a superimposed mean flow, and the results have been analyzed with the help of a simple linear finite volume model accounting for the material. Then, the model has been used to explore different combinations of geometry and material properties, with the purpose of defining design guidelines for a proper choice of the device size and the material used, that may allow fulfilling the targeted value.
\end{abstract}

\section{Introduction}

One of the current trends in the development of low emission vehicles [1] is the reduction in the car weight, so that it is becoming more and more difficult to achieve a high insulation level of the passenger compartment against the engine noise. In the case of turbocharged engines, particular problems arise [2]: on one hand, the influence on orifice noise of the waves generated by the sequence of opening and closing valves has been reduced dramatically. Indeed, both the turbo-compressor itself [3,4] and the charge air cooler [5] introduce significant damping for those low frequencies issues. On the other hand, new sources appear in the mid and high frequency range [6]. Many solutions have been proposed in order to improve quietness for mid- frequency range noise in an efficient way while avoiding any increase in pressure drop. In these solutions, with the purpose of controlling noise as close as possible to the source it is usual to include reactive silencers at the inlet and/or at the outlet of turbo-compressor, including different resonator arrangements [7, 8], interference silencers [9] and, more recently, micro-perforated materials backed by a locally reacting chamber [10].
However, in the case of supercharger or newest high pressure turbocharger noise significant sources appear at higher frequencies, which may require treatment for frequencies that can reach $10 \mathrm{kHz}$. In those specific cases, reactive silencers are useless because the waves are not propagating only as plane waves anymore and also because the required resonating volumes would be too small to be efficiently excited. The purpose of this paper is to propose and evaluate a new kind of silencer that might be able to address such high frequency issues with more complex propagating wave shape above the cross mode of the duct. By changing the mechanism of attenuation from reactive to resistive, this new solution could be actually efficient for higher frequency range without need of heavy effort for tuning. A picture of the proposed device is shown in Fig.1. The dissipative effect is attained through the use of an innovative multi-layer porous material.

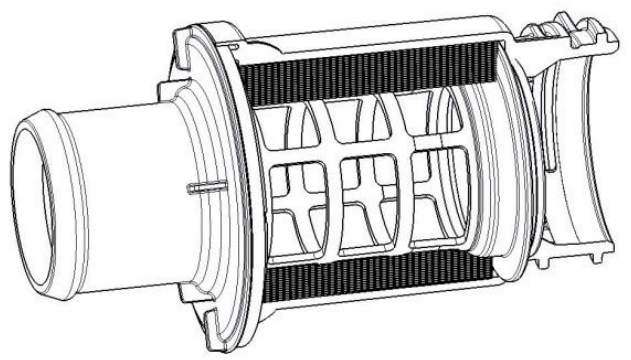

Figure 1. Scheme of the proposed device.

The paper is organized as follows: first, the experimental set-up is described and the experimental results on a first set of prototypes are discussed. Then, the models considered in the subsequent analysis are briefly described. These models are then used to explore different trends related with the geometry of the device and the properties required from the material. Finally, conclusions are drawn that may provide guidelines for the proper choice of the different design parameters.

\section{Experimental Set-up}

The experimental set-up used, together with an illustration of the measurement principle, is depicted in Fig. 2. Two main parts can be distinguished: the pulse generation system and the ducting for pulse 
propagation and measurement [11], and the mean flow supply. These two systems are briefly described in the following.

Regarding the pulse generation and propagation system, the element is excited by means of a pressure pulse generated by using an electrovalve that controls the discharge from a high pressure tank. The amplitude and the duration of the pulse can be controlled by modifying the tank pressure and the opening time of the valve in such a way that a substantially flat spectrum is achieved, thus ensuring a proper excitation. As the generated pulse propagates down the pipe (wave paths are indicated in Fig. 2 by dashed lines) instantaneous pressure is measured with piezoelectric transducers at three different points: midway between the electro-valve and the element (transducer 1), just upstream of the element (transducer 2), and downstream of the element (transducer 3). The duct lengths between the valve and the first transducer and between the third transducer and the last reflecting boundary were chosen so as to enable the isolation of the pressure pulses in the time domain at transducers 1 and 3 , and thus the pressure recorded by transducer 3 gives directly the pulse transmitted by the element.

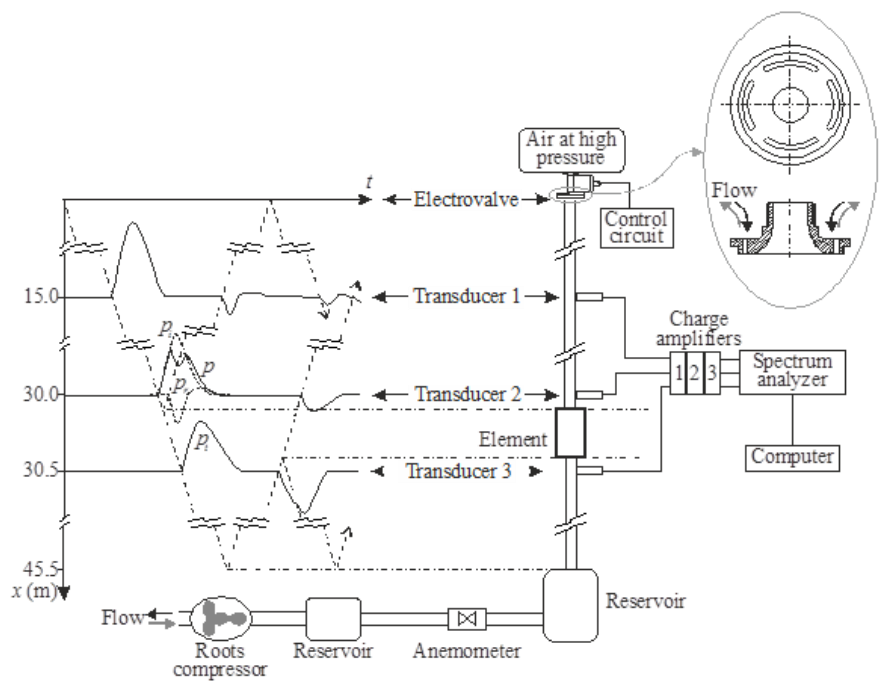

Figure 2. Scheme of the experimental set-up.

The required steady air mass flow is supplied by a roots compressor driven by an electrical motor connected to the voltage source through a frequency converter, allowing the fine control of the mass flow through the element studied. A 30 liter expansion chamber is located downstream of the compressor in order to reduce the pressure fluctuations generated by the blower. The stabilized mass flow is conveyed through a $110 \mathrm{~mm}$ diameter pipe up to a hot film anemometer, where the mass flow rate is measured. A 200 liter volume is placed at six diameters downstream of the anemometer in order to ensure stagnation conditions downstream of the flow meter. The volume outlet provides the last reflecting boundary to the pulse propagation within the ducting.

The detail in Fig. 2 shows the four ports drilled in the flange connecting the electro-valve to the propagation duct, which were designed considering the pressure drop generated, which should not be excessive for the blower, and the room available for different diameters of the propagation duct.

In order to assess the quality and representativeness of the measurements, in Fig. 3 an example of the spectrum of the incident pulse, the spectrum of the transmitted pulse and also the spectrum of the background noise signal, referred to as "threshold" in the figure, are shown. It is apparent that the incident spectrum is sufficiently flat so as to excite the device at all the frequencies. The only feature that can be observed in both the incident and the transmitted spectra is a sort of ripple close to the cut-on frequency of the first transverse mode, which appears more clearly in the transmitted spectrum. The impact of this issue on the results will be commented in the next section.

Additionally, it can be observed that the background noise is acceptably low, as it only overlaps with the transmitted signal around the frequencies just commented.

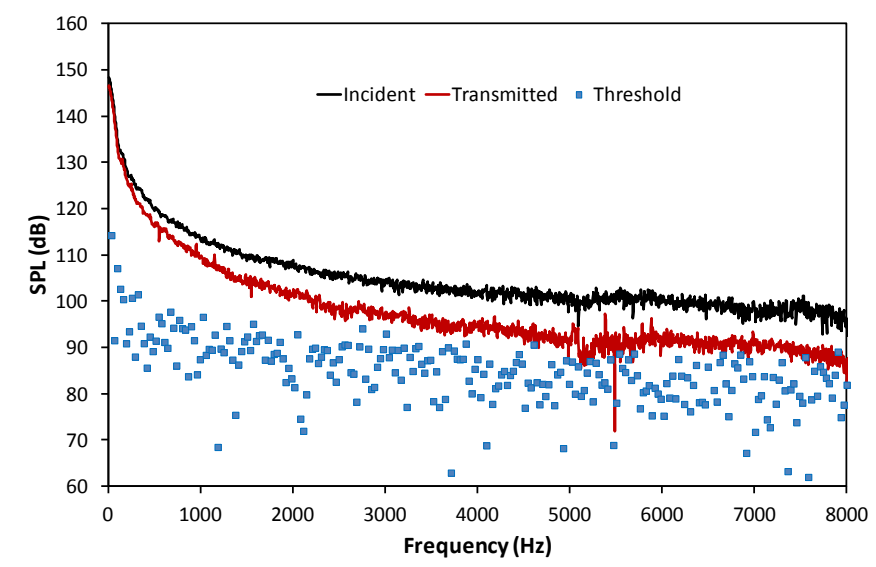

Figure 3. Comparison of incident and transmitted spectra with the threshold of the transducer.

\section{Experimental Results}

Four prototypes equipped with different porous materials were built and tested without and with a superimposed mean flow. An overall view of the results is given in Fig. 4, where the transmission loss of the four prototypes is plotted together with that of an empty device without any porous material. The expected features associated with the dissipative character of the attenuation mechanism (suppression of reactive features associated with the naked geometry, both passbands and attenuation spikes) are clearly observed for all the materials except that denoted as "material 2", in which the attenuation is consistently lower than that provided by the device without any porous material.

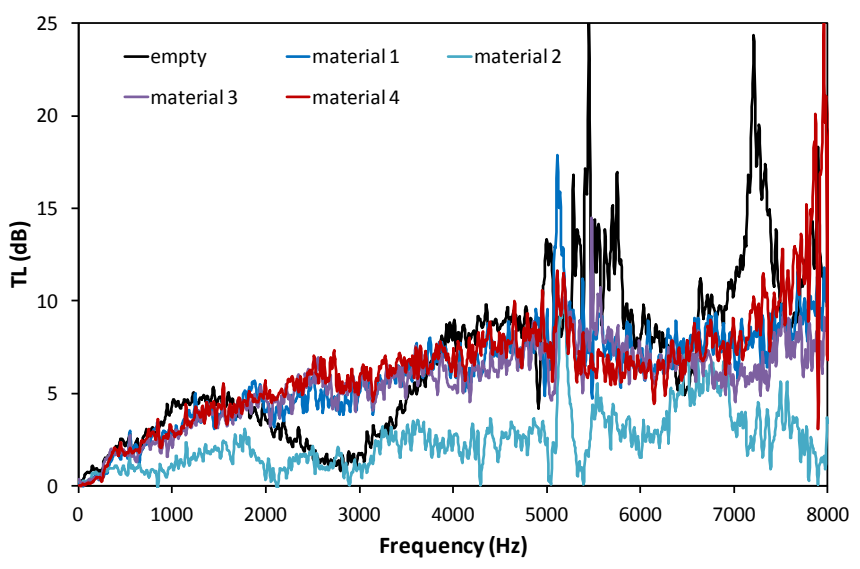

Figure 4. Transmission loss of the device: without material and with the four materials considered.

Page 2 of 8 
Apart from this, it can also be observed that, in all the cases, a very narrow spike appears around the frequency mentioned in the previous section. This strongly suggests that the measurements are contaminated locally, in this very narrow frequency band, by the excitation of the transverse mode of the propagating duct. This might be also supported by the fact that one should expect a more efficient excitation of this mode downstream of the device, due to the unavoidable non-symmetries in the actual mounting. Therefore, this particular frequency band will be obviated in all the subsequent analyses, all the more as it does not appear to affect the acoustic response at all the frequencies above and below considered in the study.

The effect of the superimposed mean flow is shown separately for the four devices considered in Figs. 5 to 8. It can be observed that, except in the case of material 2, the low to mid frequencies are not significantly affected by the presence of the flow. For high frequencies, a certain increase in the attenuation is observed for materials 1 and 3, while materials 2 and 4 do not exhibit significant changes.

In any case, one can observed that the presence of the flow does not introduce dramatic changes in the behavior of the devices. In fact, the conclusion would be that either in the presence or in absence of flow that attenuation level achieved with this starting prototypes in the high frequency range is not sufficient for any of the materials considered.

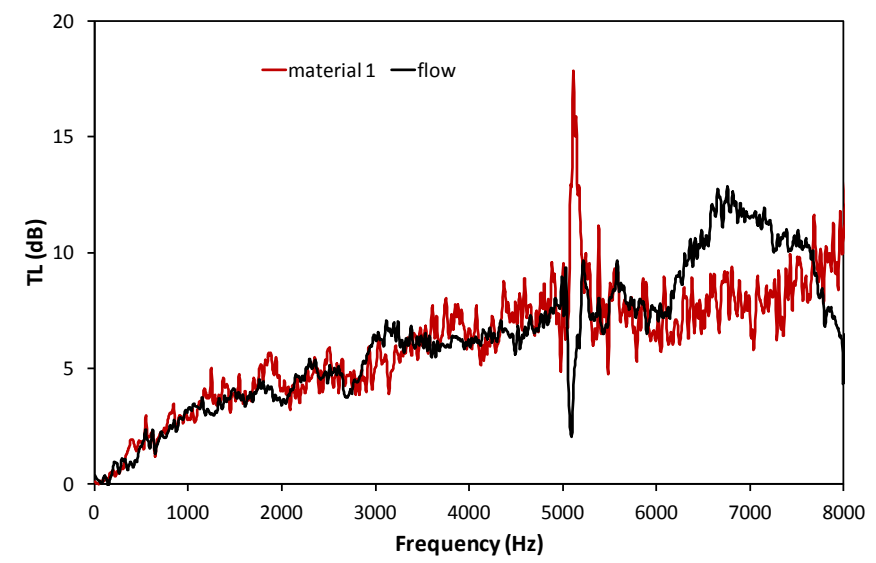

Figure 5. Effect of the flow on the transmission loss: material 1.

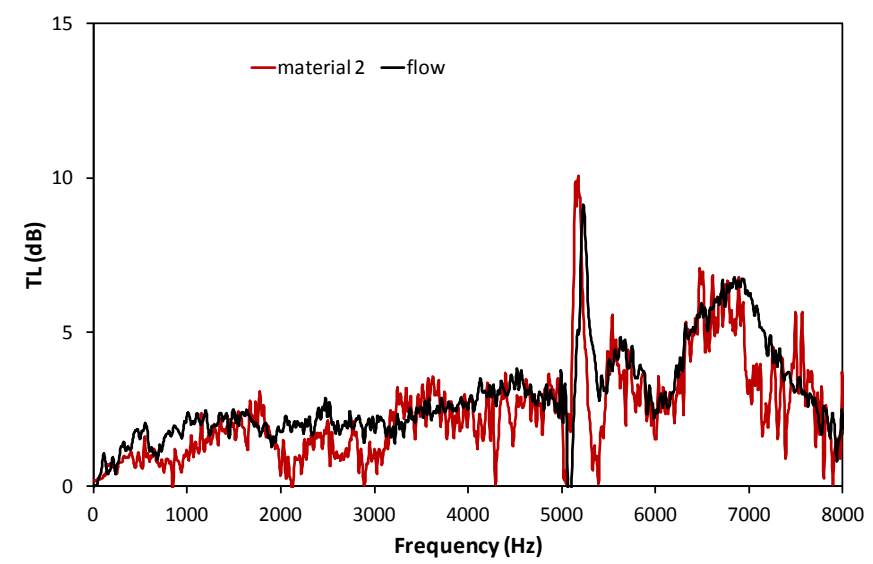

Figure 6. Effect of the flow on the transmission loss: material 2.

Page 3 of 8

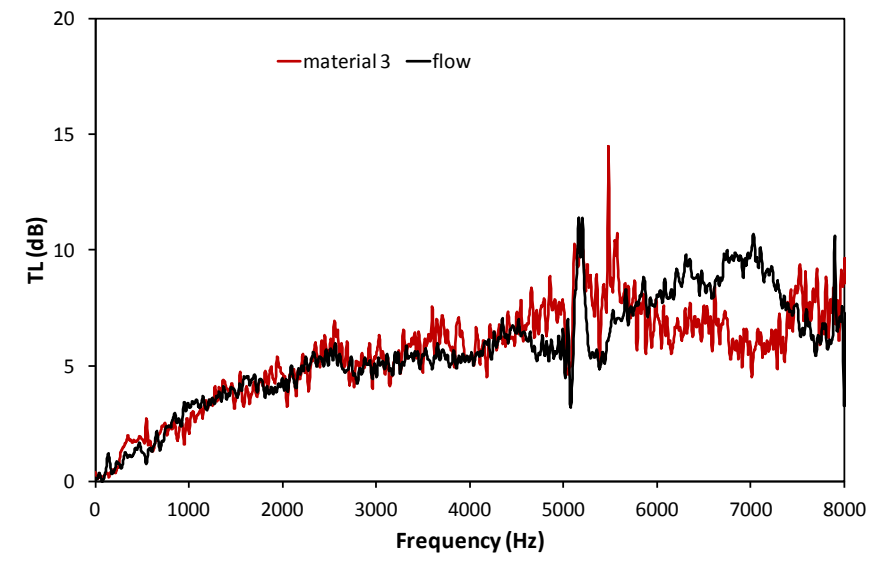

Figure 7. Effect of the flow on the transmission loss: material 3.

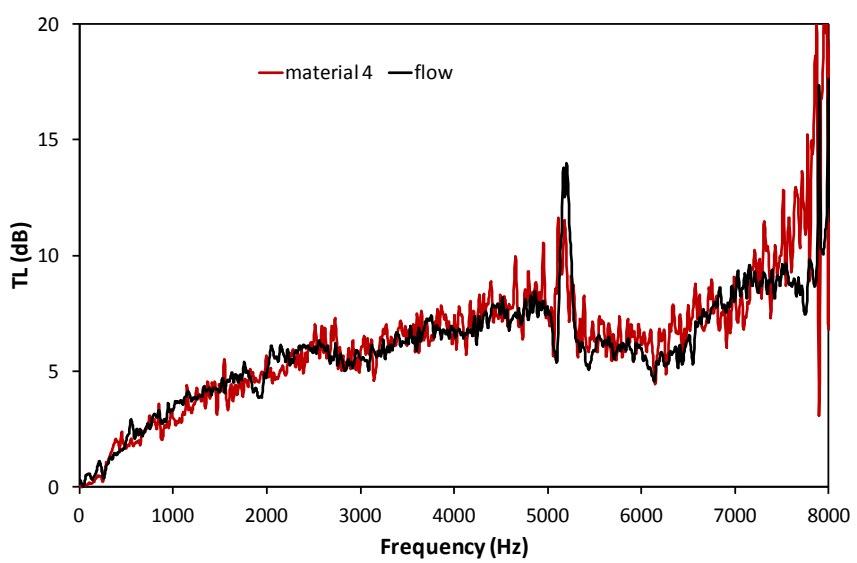

Figure 8. Effect of the flow on the transmission loss: material 4.

\section{Models}

In order to analyze the results discussed in the previous section, modelling appears to be the best option available to identify the origin of the observed features and thus to investigate possible modification to those starting configurations. In view of the observed influence of the flow, it was considered that addressing the description of the device behavior without mean flow would provide sufficiently significant insight.

Two different models were used in the study. First, an extension to absorbent materials of the linear finite volume method described in [12] and [13] was developed for the case in which, as expected, the material would behave as a bulk-reacting liner. Secondly, a transfer matrix model assuming a locally-reacting liner [14] was implemented, as the lack of information in the actual behavior of these novel materials does not permit to establish in advance which is the actual attenuation mechanism implied. The same propagation constant and impedance were assumed for the material in both cases.

The expressions used for the material characteristics and the basic formulation of the two models used are described in the following paragraphs.

\section{Material Characteristics}

Among the different possibilities available in order to describe the acoustic behavior of porous materials, models based on the specific 
flow resistivity $\sigma$ are specially convenient for practical purposes, as the need for any detailed knowledge of the material microstructure is avoided. Of course, the empirical character of most of the models available may create some trouble when considering novel materials and high frequencies, as in the case of this study.

Many models have been proposed in the literature, but those most relevant in practice were compared, in the case of porous materials, in [15], against measurements of the sound absorption coefficient. The best results were obtained making use of a new reformulation of the method proposed by Allard and Champoux [16] which was thus used here as a first reference. In the formulation proposed in [15], the non-dimensional complex characteristic impedance of the material is determined by

$$
\tilde{Z}_{c}=1+0.0729 \xi^{0.66228}-j 0.187 \xi^{0.5379}
$$

and the non-dimensional complex specific propagation constant is determined using:

$$
\tilde{k}_{c}=1+0.0982 \xi^{0.685}-j 0.228 \xi^{0.526}
$$

where $\xi=\sigma /\left(\rho_{0} f\right), f$ being the frequency and $\rho_{0}$ the air density.

However, in view of the lack of information available, it was considered convenient to use also a model based on a different correlation variable, such as that proposed by Komatsu [17], whose expressions for the non-dimensional complex characteristic impedance and the non-dimensional complex specific propagation constant are as follows:

$$
\begin{aligned}
& \tilde{Z}_{c}=1+0.00027 \xi^{6,2}-j 0.0047 \varsigma^{4.1} \\
& \tilde{k}_{c}=1+0.0004 \xi^{6.2}-j 0.0069 \xi^{4.1}
\end{aligned}
$$

where $\varsigma=2-\log (f / \sigma)$.

\section{Model with Bulk-Reacting Material}

In the frame of the finite volume calculation described in [13], a given region is regarded as built of smaller volumes, and a staggered mesh system is used, i.e. a one-dimensional momentum equation is solved at the interfaces between volumes. It can then be shown that the linearized mass and momentum equations may be interpreted in terms of volume-related junctions connected by means of length- and area-related equivalent 2-ports. The behavior of a junction regarding mass conservation is then represented by considering a multi-port with a passive impedance given by:

$$
Z^{(n)}=j \frac{a_{0}^{2}}{\omega V}
$$

where $\omega$ is the angular frequency and $a_{0}$ is the speed of sound, and which is the impedance corresponding to the compliance $V / a_{0}^{2}$ of a cavity of volume $V$. It is also shown that the linearized momentum equation, assuming harmonic time dependence for all the fluctuating variables, gives:

Page 4 of 8

$$
j \omega \frac{l}{A} v=-\Delta p
$$

where $v$ is the mass velocity, $l$ a characteristic length and $A$ the equivalent section, and which obviously admits a simple representation in terms of a 2-port, i.e., a system relating the state variables of two different points. By identifying such points with the volumes themselves, one concludes that momentum exchange at the interface between two volumes can be actually described by a 2-port defined by equation (6), which corresponds to the impedance associated with the lumped inertance $(l / A)$ of a short duct, and whose transfer matrix is given by:

$$
\left[\begin{array}{cc}
1 & j \omega(l / A) \\
0 & 1
\end{array}\right]=\left[\begin{array}{cc}
1 & j Y_{0} k_{0} l \\
0 & 1
\end{array}\right]
$$

Here, $Y_{0}$ represents the characteristic impedance in air and $k_{0}=\omega / a_{0}$ is the propagation constant.

The natural extension of the method for the inclusion of the effect of a porous material is achieved by adding a term related with the flow resistivity to the momentum equation. After linearization, this implies that the characteristic impedance and propagation constant appearing in equation (7) should be replaced by those corresponding to the porous material under study. Therefore, the transfer matrix for an equivalent two-port corresponding to the region filled with porous material is given by:

$$
\left[\begin{array}{cc}
1 & j\left(Y_{0} \tilde{Z}_{c}\right)\left(k_{0} \tilde{k}_{c}\right) l \\
0 & 1
\end{array}\right]
$$

with $\tilde{Z}_{c}$ and $\tilde{k}_{c}$ given by equations (1) and (2) or (3) and (4), respectively, depending on the model considered.

\section{Model with Locally-Reacting Material}

A transfer matrix for the propagation in the axial direction of a duct with locally-reacting liner was constructed following the ideas described in [14]. Of course, only plane wave propagation is considered, and the resulting transfer matrix is formally identical to that of a simple straight duct with rigid walls, but the effect of the liner appears in the propagation constant and the characteristic impedance. The non-dimensional impedance of the liner, for a duct radius $r$ and assuming a thickness $d$, can be approximated by

$$
\tilde{Z}_{w}=-j \tilde{Z}_{c} \cot \left(k_{0} \tilde{k}_{c} d_{e f f}\right)
$$

where $d_{\text {eff }}=d(1+d / 3 r)$, and it has been assumed that the outer wall duct is perfectly rigid and that there is no air gap between the liner and the outer wall.

The compatibility condition for the propagation constant $k$ is:

$$
k=\left(k_{0}^{2}-k_{r}^{2}\right)^{1 / 2}
$$

and $k_{r}$ is obtained by setting the non-dimensional impedance at the liner surface to the value given by equation (9), which gives 


$$
\frac{1}{U} \equiv \frac{\tilde{Z}_{w}}{k_{0} r}=j \frac{J_{0}\left(k_{r} r\right)}{k_{r} r_{0} J_{1}\left(k_{r} r\right)}
$$

An approximate solution based on a continued fractions expansion of the ratio of the Bessel functions and that is sufficient for all types of linings except in the neighbourhood of the first branch point is [14]:

$$
\left(k_{r} r_{0}\right)^{2}=\frac{96+30 j U \pm \sqrt{9216+2304 j U+912(j U)^{2}}}{12+j U}
$$

where the appropriate sign of the radical is that yielding the lowest imaginary part of $k$ when substituted in equation (10).

\section{Modelling and analysis}

\section{Model check}

As a first check of the overall suitability of the models developed, in Fig. 9 comparison is given between the measurements on the empty device and the results of the model with bulk-reacting liner when no porous material is considered. It can be seen that the agreement is very good up to the onset of the first transverse mode, and for frequencies above that significant deviations are observed. However, as is likely that the effect of the porous material will be the dominant feature for those very high frequencies, this need not be a serious shortcoming of the model.

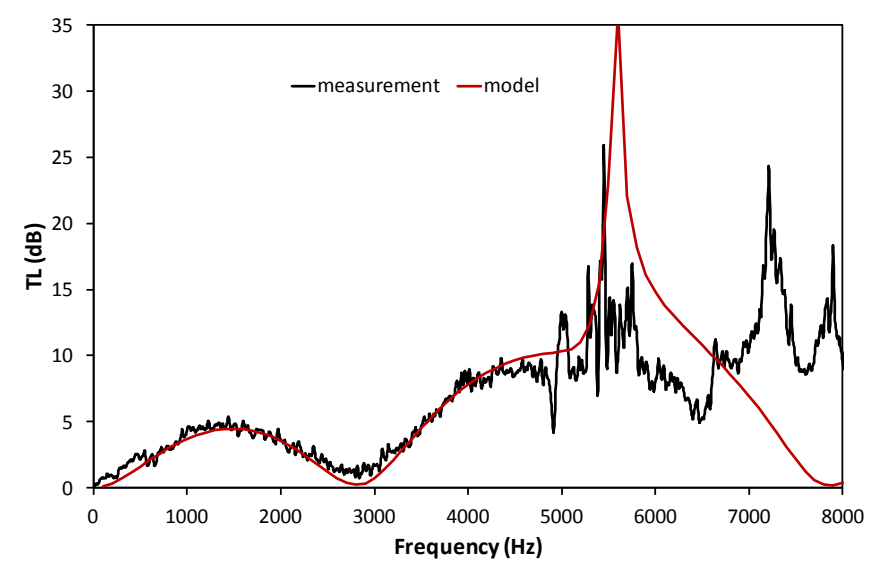

Figure 9. Comparison of model and measurement for empty device.

Now, in view of the experimental results, it is apparent that three of the materials behave in a quite similar way, and actually their flow resistivity values are of the same order of magnitude, whereas the other one (namely material 2) behaves in a quite different way, its flow resistivity being of about three times that of the other three ones. Therefore, it was considered sufficiently significant to address only material 2 and one of the other three ones (material 3). In both cases, the bulk- and the locally-reacting models were used, with the two absorbent models described in the previous section. The results are shown in Figs. 10 to 13.

As a general comment, it can be observed that regardless of the model considered, there is a certain overestimation of the attenuation at high frequencies, which could be due to the fact that the material models available deviate from the real behavior at those frequencies, at least for other materials [18].

Page 5 of 8

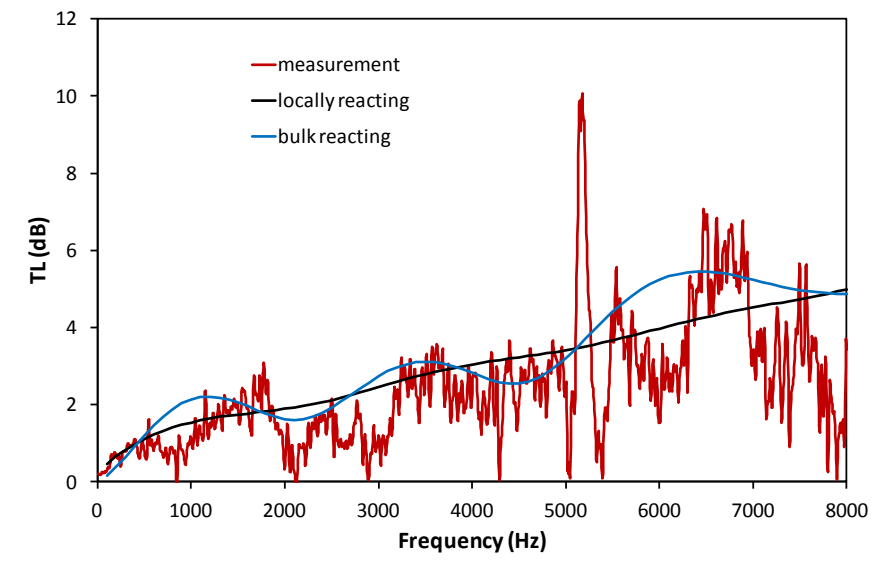

Figure 10. Material 2: comparison of bulk-reacting and locally reacting models with Allard and Champoux material model.

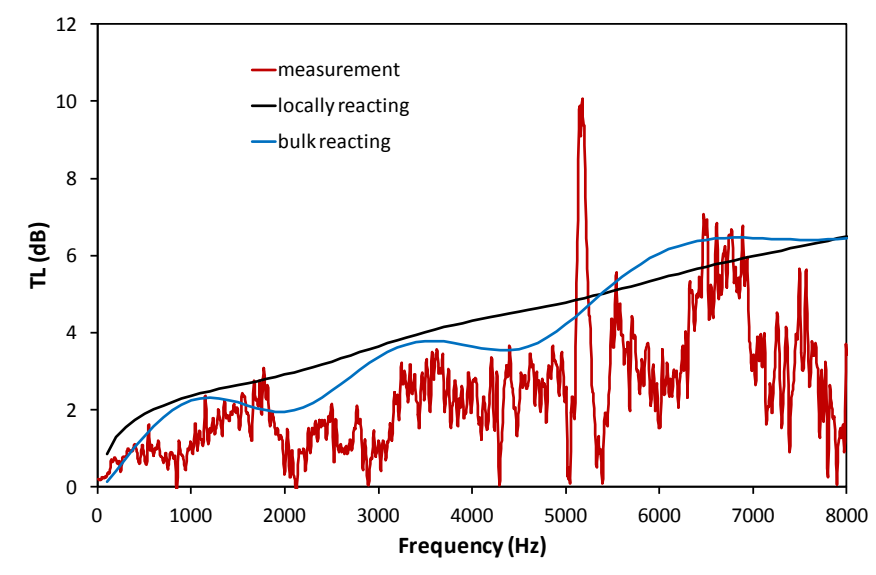

Figure 11. Material 2: comparison of bulk-reacting and locally reacting models with Komatsu material model.

Regarding the material with the highest flow resistivity, it is apparent from Figs. 10 and 11 that the model by Komatsu overestimates the attenuation, whereas the model by Allard and Champoux provides an acceptable estimate of the overall behavior, regardless of the basic approach used. actually, the attenuation here is so small that the precise shape of the measured results may not be totally significant. In any case, it is demonstrated that this extremely low attenuation is likely to be a consequence of the use of a material with an extremely high flow resistivity.

Considering now the case of material 3, shown in Figs. 12 and 13, it is apparent that now the model by Komatsu follows more closely the experimental results. Additionally, it is apparent that the locallyreacting model provides a better estimate of the low and medium frequency behavior of the device, with a less departing trend in the high frequency range. 


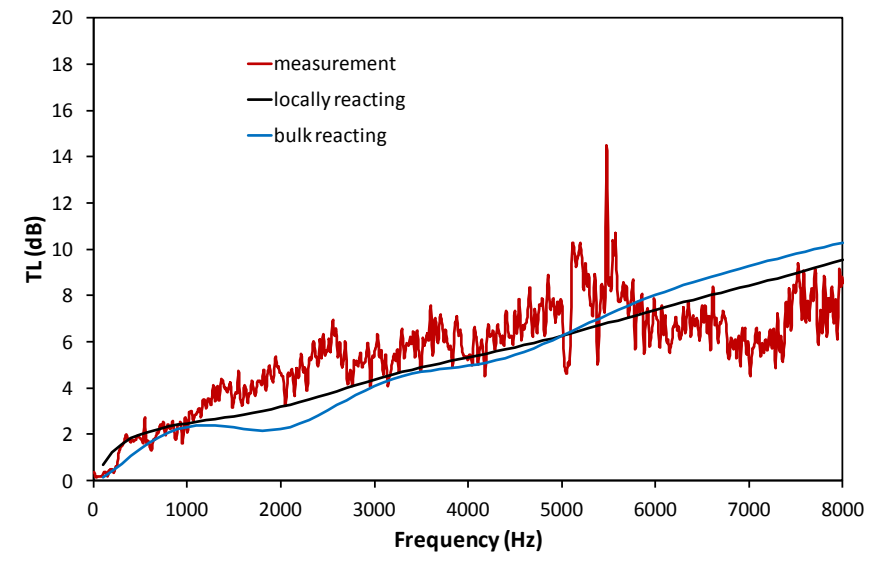

Figure 12. Material 3: comparison of bulk-reacting and locally reacting models with Allard and Champoux material model.

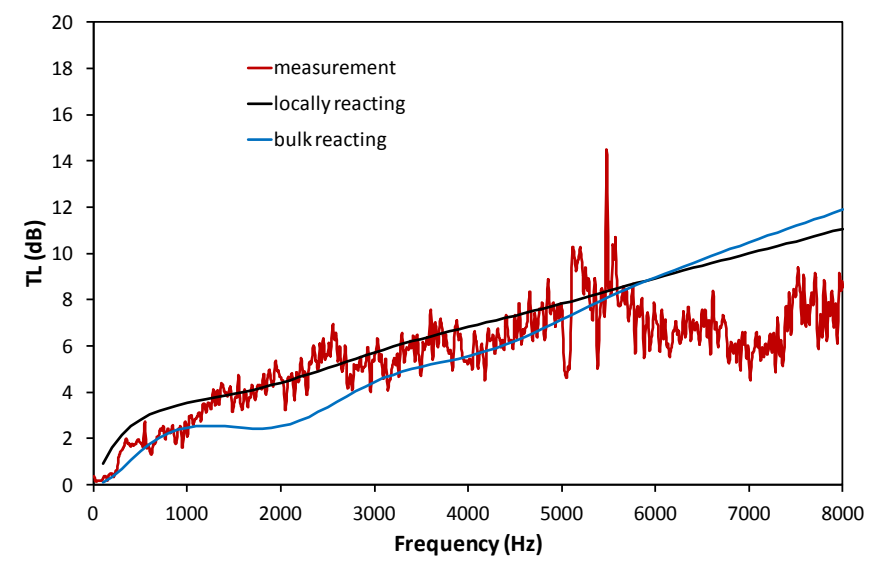

Figure 13. Material 2: comparison of bulk-reacting and locally reacting models with Komatsu material model.

As a tentative conclusion of these results, one may state that, even with the deviations observed and the substantial lack of information on the actual behavior of the precise materials used, the key point in the poor attenuation of the devices is the high value of the flow resistivity of the materials used.

\section{Analysis of trends}

In view of the previous results, it can be assumed that, at least in a qualitative sense, the models developed (and in particular the locally reacting model) may provide sensible guidelines for the definition of the material properties and the geometry of the device. This is described in the following.

\section{Effect of the flow resistivity}

Starting from the baseline value corresponding to material 3, different fractions of that value have been considered in order to analyze the effect of the flow resistivity on the performance of the device. The results obtained when a bulk-reacting liner is assumed are shown in Fig. 14; it can be observed that values in the interval between 0.15 and 0.3 of the original flow resistivity provide the best results in the high frequency range, and the value that offers a best compromise between mid and high frequencies would be around 0.15. Even so, the attenuation values attained are not high, all the more if one considers that the model (at least for very high flow resistivity) tends to overestimate the attenuation.

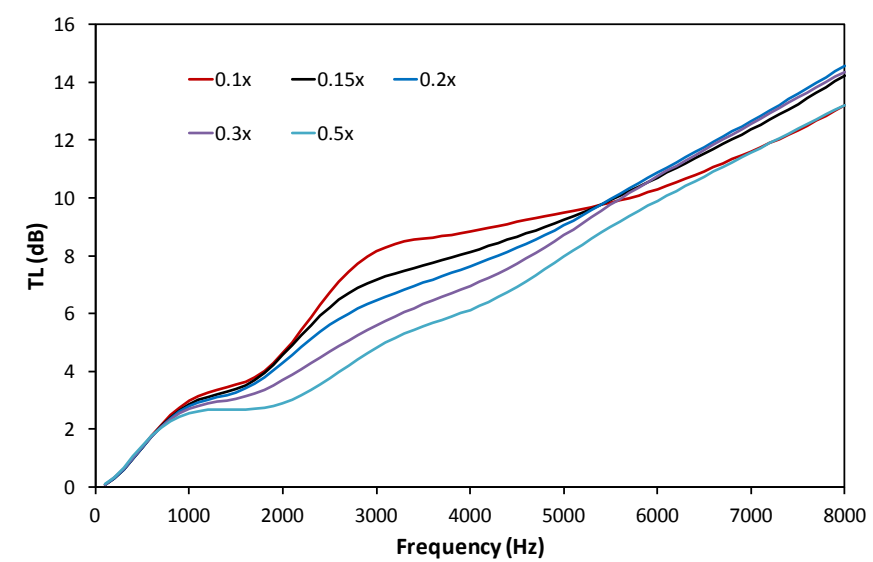

Figure 14. Effect of the flow resistivity on the transmission loss: bulk reacting model.

Therefore, a locally reacting model was also used. Obviously, depending on the particular properties of the actual material considered, the applicability of such model could require the introduction of some kind of internal partitioning in the device, so that it would be filled with rings of material layers separated by ribs. The potential benefits of such a modification (if necessary) are shown in Fig. 15, where a substantial improvement with respect to the previous case is observed. Now, it appears that the best behavior should be expected for values between 0.15 and 0.2 times the original, with suitable values at high frequencies (with all the reserve implied by the limitations of the model) and also in the typical range of whoosh noise.

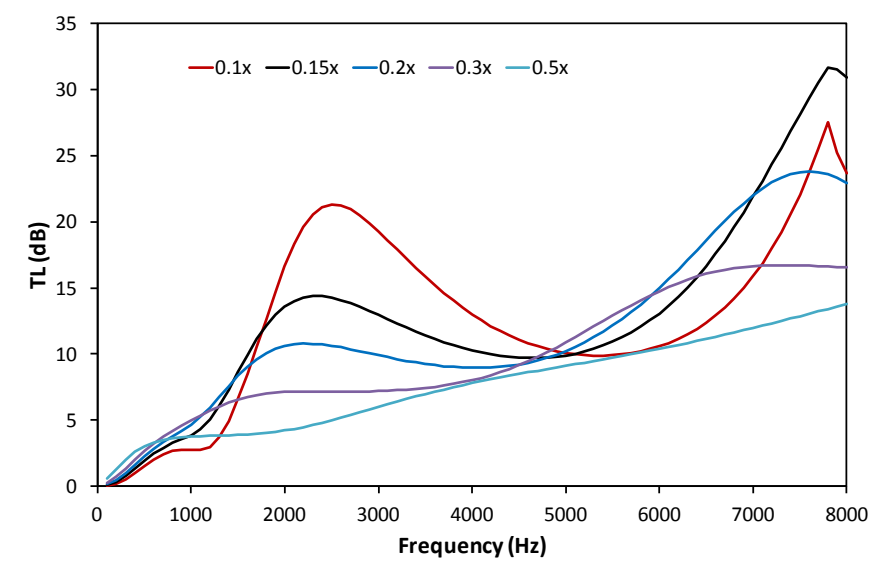

Figure 15. Effect of the flow resistivity on the transmission loss: locally reacting model.

\section{Effect of the geometry}

Given that the locally reacting assumption is that offering better perspectives, only that case has been considered when analyzing the effect of small changes in the geometry. Two different changes which do not severely affect the compactness of the device have been considered: a change in the thickness of the material, and a change in the effective axial length of the material. The flow resistivity has been kept at 0.15 times the original. 
The effect of the material thickness is illustrated in Fig. 16, where it can be observed that this can be a suitable design parameter for properly tuning the attenuation characteristics of the device. Actually, the starting value appears to be overall the best, but of course there is a clear coupling between the material and its thickness.

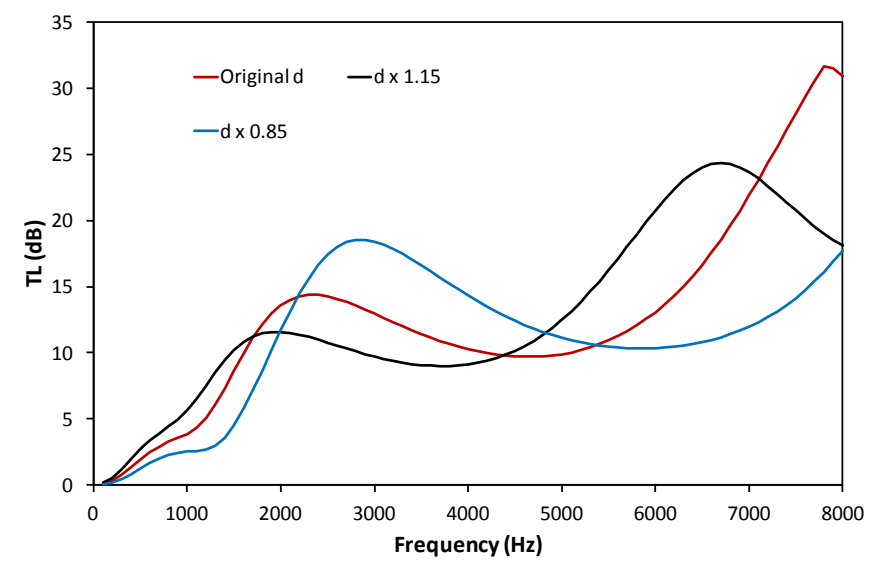

Figure 16. Effect of the material thickness on the transmission loss: locally reacting model.

The effect of the length is shown in Fig. 17. It is apparent that, again, there is a substantial influence on the behavior, but now only as an overall increase in the attenuation, without substantial changes in its shape.

Since the most difficult part of the problem lies in the design of the material, this of course provides additional degrees of freedom in the design that might minimize the exigency of a particular value of flow resistivity that may be difficult to achieve in practice.

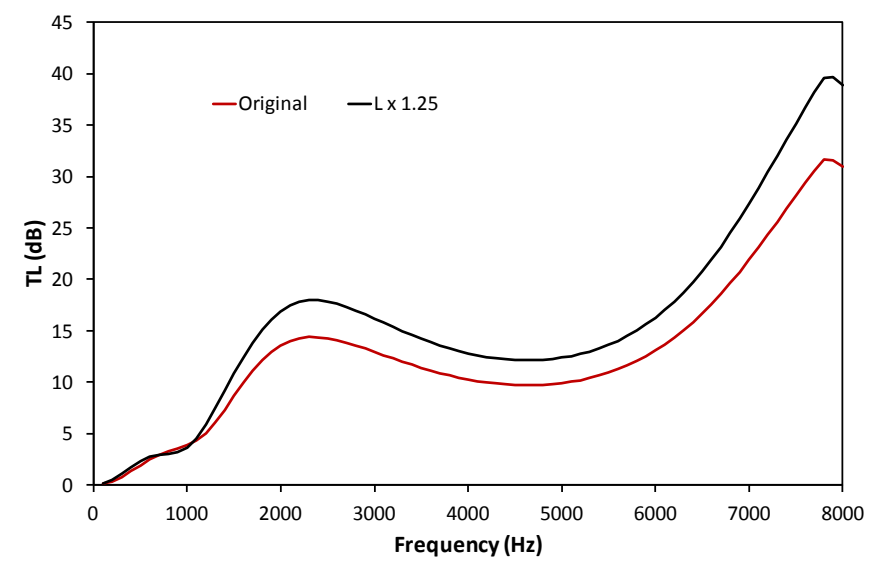

Figure 17. Effect of the material axial length on the transmission loss: locally reacting model.

\section{Summary/Conclusions}

The work presented here may be summarized as follows:

- A new compact concept for an intake silencer based on a novel multi-layer porous material has been evaluated.

- $\quad$ From the experimental work performed, it was concluded that the attenuation provided by the starting baseline prototypes is insufficient, especially in the very high frequencies for which this concept was initially conceived. Additionally, it was also checked that the influence of the mean flow was not critical.

- For the new materials, the only characteristic parameter known was the specific flow resistivity, and thus two different models, one based on a locally reacting liner and the other on a bulk reacting liner, were built in order to allow for a proper interpretation of the experimental results. Two different correlations available in the literature were considered for the description of the effect of the material.

- $\quad$ From the model results, even with the unavoidable uncertainties associated with the material behavior, it could be concluded that the main limitations of the concept come from the use of materials with an excessively high flow resistivity.

- On that basis, the models were used to explore possible design changes to improve the attenuation level. As the main outcome of this study, it was concluded that forcing a locally reacting behavior, either by the own design of the material or by its partitioning, together with a suitable choice of value of the flow resistivity, seems to be the most promising direction. Additionally, material thickness and device length provide additional degrees of freedom that may allow for the fine tuning of the product.

- However, the arguments supporting these conclusions are mostly qualitative and serious uncertainties remain mostly in the very high frequencies. Therefore, further work is needed in the full characterization of the acoustic properties of these innovative materials, both in terms of the applicability of standard correlations for the characteristic impedance and the propagation constant, and of the frequency range of validity of such correlations.

\section{References}

1. Brandl , S., Graf, B. and Rust, A., "NVH Challenges and Solutions for Vehicles with Low $\mathrm{CO}_{2}$ Emission," SAE Int. J. Passeng. Cars - Mech. Syst. 5(3):1084-1090, 2012, doi:10.4271/2012-01-1532.

2. Lu, M. and Jen, M., "Gain and Loss of NVH Characteristics from Naturally Aspirated to Turbocharged Engine," SAE Technical Paper 2007-01-2282, 2007, doi:10.4271/2007-01$\underline{2282}$.

3. Torregrosa, A.J., Serrano, J.R., Dopazo, J. and Soltani, S., "Experiments on Wave Transmission and Reflection by Turbochargers in Engine Operating Conditions," SAE Technical Paper 2006-01-0022, 2006, doi:10.4271/2006-01-0022.

4. Tiikoja, H., Rämmal, H., Abom, M. and Bodén, H., "Sound Transmission in Automotive Turbochargers," SAE Technical Paper 2011-01-1525, 2011, doi:10.4271/2011-01-1525.

5. Knutsson, M. and Åbom, M., "Acoustic Analysis of Charge Air Coolers," SAE Technical Paper 2007-01-2208, 2007, doi:10.4271/2007-01-2208.

6. Teng, C. and Homco, S., "Investigation of Compressor Whoosh Noise in Automotive Turbochargers," SAE Int. J. Passeng. Cars - Mech. Syst. 2(1):1345-1351, 2009, doi:10.4271/2009-01-2053.

7. Trochon, E., "A New Type of Silencers for Turbocharger Noise Control," SAE Technical Paper 2001-01-1436, 2001, doi:10.4271/2001-01-1436.

8. Lee, I., Selamet, A., Kim, H., Kim, T. et al., "Design of a MultiChamber Silencer for Turbocharger Noise," SAE Int. J. Passeng. Cars - Mech. Syst. 2(1):1339-1344, 2009, doi:10.4271/2009-012048.

9. Graefenstein, A. and Wenzel, W., "“Herschel-Quincke Spiral” A New Interference Silencer," SAE Technical Paper 2003-011722, 2003, doi:10.4271/2003-01-1722.

Page 7 of 8 
10. Kabral, R., Du, L., Åbom, M., and Knutsson, M., "A Compact Silencer for the Control of Compressor Noise," SAE Int. J. Engines 7(3):1572-1578, 2014, doi:10.4271/2014-01-2060.

11. Payri, F., Desantes, J.M. and Broatch, A., "Modified Impulse Method for the Measurement of the Frequency Response of Acoustic Filters to Weakly Nonlinear Transient Excitations, $J$. Acoust. Soc. Am. 107:731-738, 2000.

12. Amphlett, S., Niven, P., Payri, F., and Torregrosa, A., "Linear Acoustic Modelling using 1-D Flow Systems which represent Complex 3-D Components," SAE Technical Paper 2011-011524, 2011, doi:10.4271/2011-01-1524.

13. Torregrosa, A.J., Broatch, A., Gil, A. and Moreno, D.,"Analysis of Acoustic Networks including Cavities by means of a Linear Finite Volume Method, J. Sound Vib. 331: 4575-4586, 2012, doi:10.1016/j.jsv.2012.05.023.

14. Frommhold, W. and Mechel, F.P., "Simplified Methods to Calculate the Attenuation of Silencers," J. Sound Vib. 141:103125, 1990, doi:10.1016/0022-460X(90)90516-3.

15. Oliva, D. and Hongisto, V. "Sound absorption of porous materials - Accuracy of prediction methods," App. Acoust. 74: 1473-1479, 2013, doi: 10.1016/j.apacoust.2013.06.004.

16. Allard J.F and Champoux Y., "New Empirical Equations for Sound Propagation in Rigid Frame Fibrous Materials," $J$. Acoust. Soc. Am.91(6):3346-3353, 1992, doi:10.1121/1.402824.

17. Komatsu, T.,"Improvement of the Delany-Bazley and Miki Models for Fibrous Sound-Absorbing Materials. Acoust. Sci. Technol. 29(2):121-129, 2008, doi:10.1250/ast.29.121.

18. Kino, N., "Further Investigations of Empirical Improvements to the Johnson-Champoux-Allard Model," App. Acoust. 96: 153170, 2015, doi: 10.1016/j.apacoust.2015.03.024.

\section{Contact Information}

Prof. Antonio J. Torregrosa

CMT - Motores Térmicos. Universitat Politècnica de València Camino de Vera s/n. 46022 Valencia (Spain). Tel. +34 963877658. e-mail: atorreg@mot.upv.es

\section{Definitions/Abbreviations}

$\begin{array}{ll}\text { A } & \text { section } \\ a_{0} & \text { speed of sound } \\ d & \text { material thickness } \\ f & \text { frequency } \\ k & \begin{array}{l}\text { effective propagation } \\ \text { constant } \\ k_{0}\end{array} \\ \tilde{k}_{c} & \begin{array}{l}\text { air propagation constant } \\ \text { non-dimensional complex } \\ \text { propagation constant }\end{array} \\ l & \text { characteristic length }\end{array}$

Page 8 of 8

$\begin{array}{ll}p & \text { pressure } \\ r & \text { radius } \\ v & \text { mass velocity } \\ Y_{0} & \text { air characteristic impedance } \\ \tilde{Z}_{c} & \text { non-dimensional complex } \\ & \text { characteristic impedance } \\ \rho_{0} & \text { air density } \\ \sigma & \text { specific flow resisitivity } \\ \omega & \text { angular frequency }\end{array}$

ngular frequency 